\section{Thyrotoxicosis in a neonate of a mother with no history of thyroid disease}

This case of thyrotoxicosis in a neonate presented a physical appearance of thyroid underactivity, whereas the signs were more in keeping with thyrotoxicosis. Biochemical evidence of thyrotoxicosis was lacking until the baby was 28 days old, and there was no history of thyroid disease in the parents or the family.

\section{Case report}

The patient, a girl, was born in May 1974 at 38 weeks' gestation after a normal pregnancy and delivery. She weighed $3.12 \mathrm{~kg}$ and the healthy placenta weighed $0.74 \mathrm{~kg}$. She was seen by one of us on the third day of life because of rectal prolapse and frequent soft stools, the $p \mathrm{H}$ of which was 6 . She was fed by breast and complementary milk formula and, though slightly loose stools continued, she gained weight. Because of coarse facial features and a flat nasal bridge, cretinism was suspected. Thyroid function tests were done on the fourth day, and serum thyroxine was within the normal range at this time (Table). It became apparent later that the baby had similar facial features to her father.

Her weight at 20 days of age was $2.88 \mathrm{~kg}$, or $0.24 \mathrm{~kg}$ below birthweight, and because of this and continuing loose stools she was admitted to this hopsital. Her facial features and rectal prolapse were unchanged, but she was constantly hungry and restless and had a staring expression and shiny eyes (Fig). The heart rate at rest was 160/min. There was no visible goitre. The serum thyroxine level was within the normal range for age (Table). Despite the absence of supporting laboratory evidence, it was thought that the diarrhoea might be due to congenital lactase deficiency. Feeds of lactose-free milk (Galactomin 17) were begun but produced no striking clinical improvement.
On the 28th day the serum thyroxine level was raised (Table), and was still raised on the 32nd and 34th days.

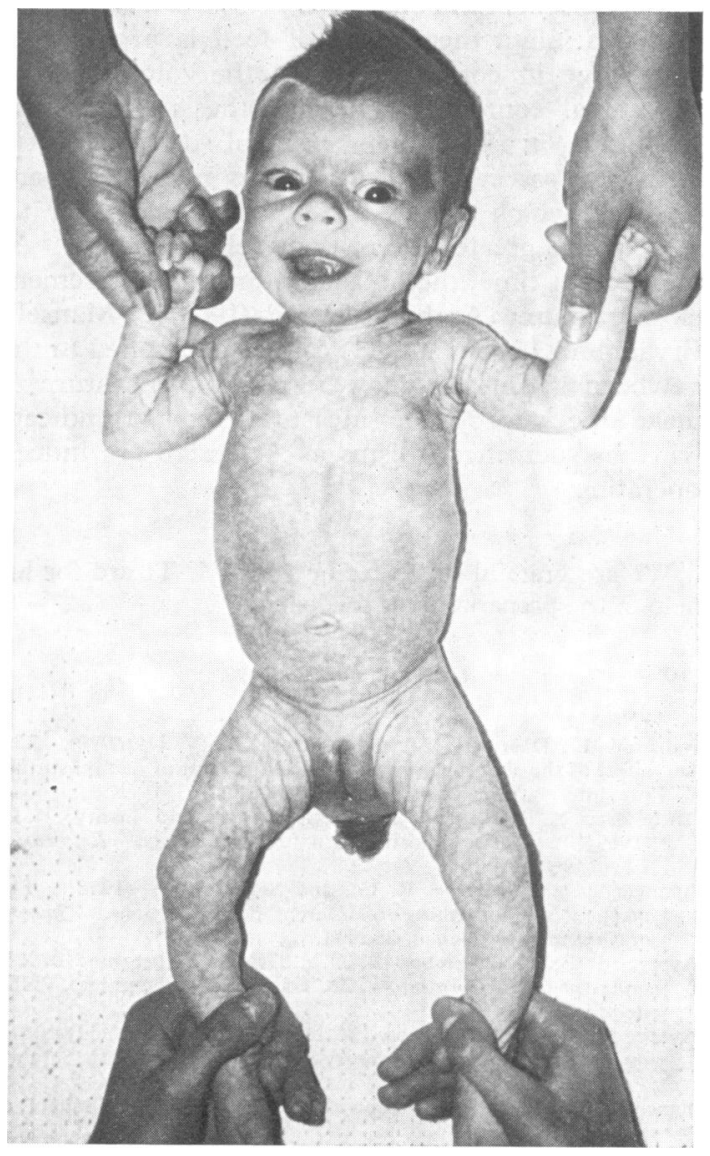

FIG.-Appearance at 3 weeks.

TABLE

Serum thyroxine $\left(T_{4}\right)$ levels $(\mu \mathrm{g} / 100 \mathrm{ml})$ in baby

\begin{tabular}{l|c|c|c|c|c|c|c|c|}
\hline & \multicolumn{2}{c}{ At age of: } \\
\cline { 2 - 5 } & $4 \mathrm{~d}$ & $24 \mathrm{~d}$ & $28 \mathrm{~d}$ & $32 \mathrm{~d}$ & $34 \mathrm{~d}$ \\
\hline $\begin{array}{c}\text { Age-related normal levels } \\
\text { (O'Halloran and Webster, 1972) }\end{array}$ & $9 \cdot 8-16 \cdot 6$ & $8 \cdot 2-16 \cdot 6$ & $8 \cdot 3-16 \cdot 3$ & $8 \cdot 3-16 \cdot 3$ & $8 \cdot 3-16 \cdot 3$ & $7 \cdot 5-16 \cdot 1$ & $6 \cdot 7-13 \cdot 9$ \\
\hline
\end{tabular}


The serum thyroid stimulating hormone (TSH) level was normal at $3 \cdot 1 \mu \mathrm{U} / \mathrm{ml}$, and the thyroglobulin antibody tests and the fluorescent antibody tests for antinuclear factor (ANF), thyroid colloid, and microsomal elements were negative. The long-acting thyroid stimulator (LATS) index was normal at $100 \%$ on the 54 th day. Other tests excluded infection and cystic fibrosis. The radiological bone age was not advanced. There had been some clinical suspicion of thyrotoxicosis and, having obtained supporting biochemical evidence, treatment was started with carbimazole $1 \mathrm{mg}$ 6-hourly. This produced gradual improvement with weight gain and diminution of appetite, overactivity, and diarrhoea. Carbimazole was gradually reduced and discontinued after 4 weeks, when the patient was clinically euthyroid. Subsequent serum thyroxine levels were within the normal range (Table.)

Good progress was maintained at home with improvement in the prolapse, but the reintroduction of a milk formula resulted in the return of diarrhoea and aggravation of the rectal prolapse. Readmission to hospital was necessary and Galactomin 17 feeds were started again. The response to a glucose tolerance test at 8 months of age was normal with a fasting glucose of 50 $\mathrm{mg} / 100 \mathrm{ml}, 115 \mathrm{mg} / 100 \mathrm{ml}$ at one hour, and $65 \mathrm{mg} / 100$ $\mathrm{ml}$ at 2 hours. A subsequent lactose tolerance test produced a rise of only $5 \mathrm{mg} / 100 \mathrm{ml}$ in blood glucose from a fasting level of $70 \mathrm{mg} / 100 \mathrm{ml}$. On the evening of the test diarrhoea restarted and continued for 12 hours. At the age of one year small quantities of cow's milk were added to the diet and when last seen she tolerated about $90 \mathrm{ml}$ daily. After the age of 6 months the rectum remained in the normal position.

Investigation of parents. The patient's mother and father were first cousins. The mother had had 2 normal children. There was no past or present history of thyroid or related disease in either parents or family. Serum thyroxine and TSH levels were normal in both parents and maternal thyroid antibody tests were negative. However, the mother's LATS index was substantially raised at $377 \%$ when the baby was 54 days' old. The mother's LATS protector when the baby was aged 7 months was normal, and also by this time, her LATS level had returned to normal. LATS was measured by mouse bioassay (Adams, 1958).

\section{Discussion}

Rectal prolapse in the newborn is usually associated with diarrhoea or a malabsorption state such as cystic fibrosis. The loose motions in our patient began in the early neonatal period at a time when lactase deficiency was a possible contributing factor.

The clinical picture was confusing. The coarse features suggested thyroid underactivity, but the restlessness, hunger, and loose motions were more in keeping with thyrotoxicosis. Until the 28th day of life there was no conclusive biochemical evidence of thyrotoxicosis.

Thyrotoxicosis in neonates is generally thought to be due to transplacental passage of LATS (Rosenberg, Grand, and Silbert, 1963) or of LATS protector substance (Nutt et al., 1974). The symptoms are usually evident shortly after birth but can be delayed by antithyroid treatment of the mother. The discrepancy between the symptoms and the initially normal thyroid function tests in our patient are difficult to explain. The transient nature of the condition, bowever, accords with the usual clinical course, which is due to the 30-day half life of maternal LATS antibody (McKenzie, 1964). We can only speculate on the role of TSH transmitted in breast milk to this baby, who was breastfed until the 20th day of life.

A raised LATS index may be found in $21 \%$ of relatives of patients with thyroid disease (Wall, Good, and Hetzel, 1969). The mother of our patient had no history of thyroid disease nor was any history of it in the family. Possibly the mother will become hyperthyroid. To our knowledge no one has monitored the LATS index throughout pregnancy in normal mothers. Perhaps an anamnestic reaction occurred in the mother in our case and it caused temporary neonatal thyrotoxicosis in her child.

We attribute the normal LATS activity in the baby to it being measured too late (54th day) to catch it at its peak.

An alternative but less likely explanation for the baby's hyperthyroid condition is that it was genetic. Hollingsworth, Mabry and Eckerd, (1972) thought the mechanism might be either mother/daughter inheritance or a recessive trait brought out by a consanguineous marriage, as in our case.

The evidence in favour of congenital deficiency of lactase is strong but not complete. This in itself would account for a restless baby with loose stools, and would be aggravated in our patient by the increased intestinal motility associated with hyperthyroidism. Lactase deficiency is probably genetically determined and in our case an autosomal recessive mechanism would be tenable because of the parents' consanguinity.

This case shows show a definitive diagnosis may be delayed in an illness when two unusual conditions are contributing to the symptoms. Temporary thyrotoxicosis must now be considered in neonates who fail to thrive even in the absence of a history of maternal thyroid disease.

\section{Summary}

A newborn infant had rectal prolapse, congenital lactase deficiency, and temporary neonatal thyrotoxicosis. The thyrotoxicosis was associated with a raised long-acting thyroid stimulator index in a 
mother with no personal or family history of thyroid or related autoimmune disease. The parents were first cousins.

We thank Mr. R. Cudmore for surgical help, and Dr. S. M. Dirmikis, of the University of Sheffield, for the LATS protector measurement.

\section{REFERENCES}

Adams, D. D. (1958). The presence of an abnormal thyroid stimulating hormone in the serum of some thyrotoxic patients. Fournal of Clinical Endocrinology and Metabolism, 18, 699.

Hollingsworth, D. R., Mabry, C. C., and Eckerd, J. M. (1972). Hereditary aspects of Graves' disease in infancy and childhood. Fournal of Pediatrics, 81, 446.

McKenzie, J. M. (1964). Neonatal Graves' disease. Fournal of Clinical Endocrinology and Metabolism, 24, 660.

Nutt, J., Clark, F., Welch, R. G., and Hall, R. (1974). Neonatal hyperthyroidism and long-acting thyroid-stimulator protector. British Medical fournal, 4, 695.

O'Halloran, M. T., and Webster, H. L. (1972). Thyroid function assays in infants. Fournal of Pediatrics, 81, 916.

Rosenberg, D., Grand, M. J. H., and Silbert, D. (1963). Neonatal hyperthyroidism. New England fournal of Medicine, 268, 292.

Wall, J. R., Good, B. F., and Hetzel, B. S. (1969). Long-acting thyroid stimulator in euthyroid relatives of thyrotoxic patients. Lancet, 2, 1024.

D. S. K. Brookfield, ANNE E. McCandless ${ }^{\star}$, and C. S. SMITH

Department of Child Health, Alder Hey Children's Hospital, Liverpool.

${ }^{\star}$ Correspondence to Dr. A. E. McCandless.

\section{Neonatal brain growth during prolonged intravenous feeding}

Total intravenous feeding may occasionally be a life-saving procedure for the seriously ill low birthweight infant. The technical and metabolic complications associated with total intravenous nutrition have been well reviewed (Harries, 1971; Harries, 1972; Heird et al., 1972; Heird and Winters, 1975; Baum and Aynsley-Green, 1975). However, little is known about the effects of prolonged intravenous feeding on tissue growth and development in the newborn. In particular it would be important to know the effects of longterm intravenous nutrition on the brain, since in the neonatal period when the brain is growing rapidly it may be susceptible to nutritional deprivation (Dobbing, 1974).

Dobbing and Sands (1973) have provided standards for the biochemical development of the neonatal brain by analysing brains ranging in age from 10 weeks' gestation to 7 postnatal years. Using these standards for comparison, we have attempted to assess the effects of long-term intravenous nutrition on the growth and development of the brain from an infant who was born at 32 weeks' gestation and who died after 10 weeks of continuous intravenous feeding.

\section{Case report}

A male infant weighing $1270 \mathrm{~g}$ was born at 32 weeks of gestation. He required assisted ventilation from birth and for the next 7 weeks, initially on intermittent positive pressure ventilation and subsequently on continuous distending airways pressure. During the first few days the infant had delayed emptying of the stomach. On day 5 he was started on total intravenous feeding using a regimen of Vamin (fructose) and Intralipid (Baum et al., 1975). During the second week of life there was abdominal distension and clinical evidence of intestinal obstruction. $X$-ray of the upper gastrointestinal tract, using gastrografin contrast material, showed obstruction at the level of the jejunum and at the ileocaecal valve. Intravenous feeding was continued. Over the next 7 weeks there was evidence of persistant intestinal obstruction but the infant was too ill for a laparotomy to be performed. At 9 weeks' postnatal age a gastrografin enema showed the presence of a jejunocolic fistula. His respiratory status had improved sufficiently for an exploratory operation to be performed which showed multiple fistulae and perforations throughout the jejunum, ileum, and caecum. $10 \mathrm{~cm}$ of jejunum were resected and a colostomy constructed. Postoperatively the colostomy did not function and there was further evidence of fistula formation; at a postnatal age of 10 weeks (gestational age 42 weeks) he died of faecal peritonitis.

Details of the intravenous feeds which this infant received are given in Table $I$. For most of his life he received $100 \mathrm{cal} / \mathrm{kg}$ per 24 hours, $4 \mathrm{~g}$ of fat as intralipid/kg per 24 hours, and $3 \mathrm{~g}$ amino acids/kg per 24 hours. In addition, attempts were made to maintain homoeostasis for electrolytes, vitamins, and trace minerals. The most serious metabolic problem documented was hypophosphataemia, which was corrected by the addition of increased phosphate supplements and has been reported elsewhere (Woods et al., 1974).

Growth on intravenous feeding. The infant received continuous intravenous feeding from day 5 after birth until death 10 weeks later, during which time there was a strong clinical impression of increase in length and, to a limited extent, in muscle bulk. Unfortunately no clinical measurements of length nor of limb girth were made. Birthweight was $1270 \mathrm{~g}$, which is on the 10th centile for 32 weeks, and at necropsy weight was $1680 \mathrm{~g}$, below the 3rd centile for 42 weeks' gestation. The occipitofrontal head circumference at birth was $29 \mathrm{~cm}$, on the 50 th centile for 32 weeks, whereas the post-mortem head circumference was $33 \mathrm{~cm}$, 10th centile for 42 weeks' gestation (standards from Davies et al., 1972).

Measurement of brain growth. A post-mortem examination was performed on the day the infant died. 